\title{
Sugammadex associated profound bradycardia and sustained hypotension in patient with the slow recovery of neuromuscular blockade - A case report -
}

Received October 22, 2018

Revised November 26, 2018

Accepted December 2, 2018

\section{Corresponding author}

Ki Tae Jung, M.D., Ph.D.

Department Anesthesiology and Pain Medicine, Chosun University Hospital, 365 Pilmun-daero, Donggu, Gwangju 61453, Korea

Tel: 82-62-220-3223

Fax: 82-62-223-2333

E-mail: mdmole@chosun.ac.kr ORCID

https://orcid.org/0000-0002-2486-9961

\section{Yong Jun Choi, Jeong Wook Park, Sang Hun Kim, and Ki Tae Jung}

Department of Anesthesiology and Pain Medicine, Chosun University Hospital, Gwangju, Korea

Background: New complications associated with sugammadex have been increased since its widespread use. We report a case of an 80-year-old male who experienced profound bradycardia and sustained hypotension after administration of sugammadex.

Case: Following administration of 200 mg sugammadex after laparoscopic cholecystectomy, sudden bradycardia (29 beats/min) developed for 10 seconds and his trainof-four (TOF) ratio remained at 0.2 for $5 \mathrm{~min}$. An additional $200 \mathrm{mg}$ sugammadex was administered and profound bradycardia (21-30 beats/min) and hypotension (60/40 $\mathrm{mmHg}$ ) developed. Atropine at $0.5 \mathrm{mg}$ was administered, but the effect lasted only 30 $\mathrm{s}$. Profound bradycardia occurred four more times at $30 \mathrm{~s}$ intervals, and ephedrine and phenylephrine were injected intermittently to increase the patient's heart rate and blood pressure. The TOF ratio became 0.9 about 10 min after administration of additional sugammadex.

Conclusions: Awareness must be heightened regarding the possibility of sugammadexinduced bradycardia and hypotension, and more attention should be paid to patients with slow recovery times following muscle relaxation, despite the use of sugammadex.

Keywords: Bradycardia; Hypotension; Neuromuscular blockade; Postoperative complications; Sugammadex.
Since sugammadex was introduced in the clinical field, it has been widely used as a rapid and relatively safe reversal drug for neuromuscular blocking agents. However, lifethreatening side effects, such as hypersensitivity reactions have been reported [1]. Hypersensitivity-associated complications were reported prior to approval by the United States Food and Drug Administration (FDA). Since more widespread use of sugammadex, new complications such as hypotension, QT prolongation, and bradycardia have been reported that anesthesiologists should be aware of it [2]. Recently, there have been a few reports of cases of severe brady- cardia associated with sugammadex [3-6]. Here, we report a case of profound bradycardia and sustained hypotension in a patient with slow recovery from neuromuscular blockade, despite administration of sugammadex.

\section{CASE REPORT}

An 80-year-old male patient (height: $165 \mathrm{~cm}$, weight: $75 \mathrm{~kg}$ ) was scheduled for laparoscopic cholecystectomy surgery for acute cholecystitis. He had a past history of hypertension and diabetes mellitus, but they were well controlled with medica-

This is an Open Access article distributed under the terms of the Creative Commons Attribution Non-Commercial License (http://creativecommons.org/licenses/by-nc/4.0) which permits unrestricted non-commercial use, distribution, and reproduction in any medium, provided the original work is properly cited. 
tions ( $\beta$-blocker, aspirin, metformin). Preoperative laboratory tests showed chronic kidney disease (stage 3) with increased blood urine nitrogen $(28.9 \mathrm{mg} / \mathrm{dl})$ and creatinine $(1.35 \mathrm{mg} /$ dl), but electrolyte results were within normal limits (Na, 142 $\mathrm{mEq} / \mathrm{L} ; \mathrm{K}, 3.6 \mathrm{mEq} / \mathrm{L}$ ). His liver enzymes were increased (aspartate aminotransferase, $136.8 \mathrm{mg} / \mathrm{dl}$; alanine aminotransferase, $276.4 \mathrm{mg} / \mathrm{dl}$ ) due to acute cholecystitis. Arterial blood gas analysis (ABGA) results were as follows: $\mathrm{pH}$ 7.473, $\mathrm{PaO}_{2}$ 62.0 mmHg, $\mathrm{PaCO}_{2} 25.7 \mathrm{mmHg}, \mathrm{SaO}_{2} 93.6 \%$, and $\mathrm{HCO}_{3} 21.6$ $\mathrm{mM}$. Electrocardiography showed normal sinus rhythm with 1st degree atrioventricular (AV) block and QT/corrected QT (QTc) of 418/454 ms. Physical examination was unremarkable.

The patient was administered midazolam $(0.05 \mathrm{mg} / \mathrm{kg}$, intramuscularly) $30 \mathrm{~min}$ before anesthesia and transferred to the operating room. Standard monitoring was performed with electrocardiogram (ECG), pulse oximetry $\left(\mathrm{SpO}_{2}\right)$, noninvasive blood pressure (BP), and bispectral index (BIS moni- tor A-2000, Aspect Medical Systems, USA), and NMT sensor (888418 M-NMT MechanoSensor, Datex-Ohmeda Inc., Finland). The monitored values were recorded during the operation using data acquisition software (Vital Recorder, VitalDB, Korea). Preoperative hemodynamic values were as follows: $\mathrm{BP}$ 104/53 mmHg, heart rate (HR) 66 beats/min, $\mathrm{SpO}_{2} 98 \%$. Anesthesia was induced and maintained by total intravenous anesthesia using propofol and remifentanil to maintain the BIS score within the 40 to 60 range. Endotracheal intubation was performed after achievement of adequate neuromuscular blockade by rocuronium bromide $(0.6 \mathrm{mg} / \mathrm{kg})$. Neuromuscular function was assessed with TOF stimuli during the operation. After the induction of anesthesia, radial arterial cannulation was done, and arterial BP was monitored during the surgery.

The patient's BP and HR remained stable during the surgery, but surgery took almost $3 \mathrm{~h}$ because of severe adhesion of intraperitoneal organs due to inflammation. An additional

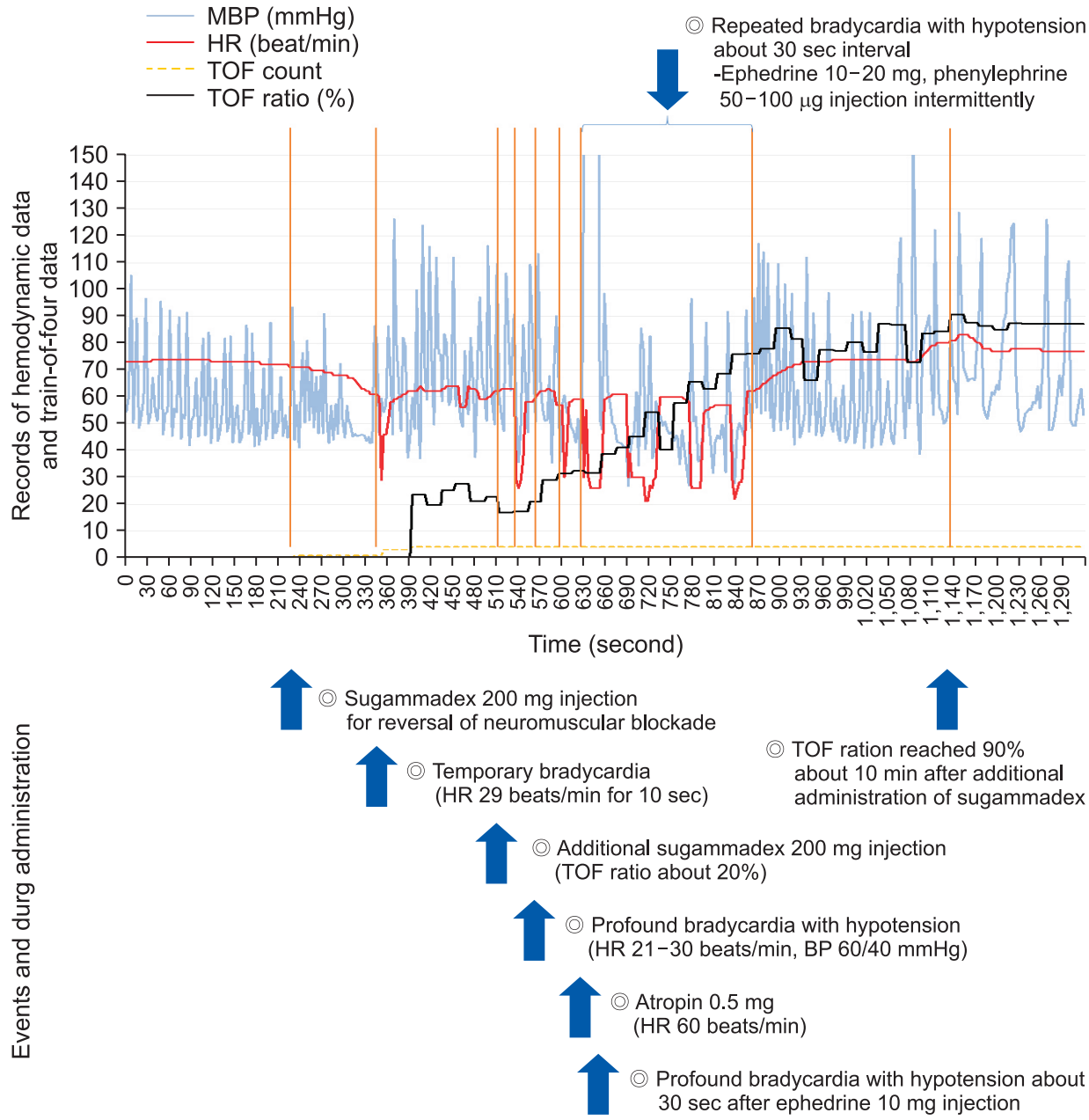

Fig. 1. Hemodynamic data and trainof-four (TOF) data of the patient. Bradycardia has developed after use of sugammadex shortly. Reversal from neuromuscular blockade was inadequate despite using sugammadex. Profound bradycardia and sustained hypotension were developed after administration of an additional dose of sugammadex. Adequate reversal from neuromuscular blockade was achieved about 10 min after administration of additional use of sugammadex. MBP: mean blood pressure, HR: heart rate, BP: blood pressure. 
dose of rocuronium $10 \mathrm{mg}$ was administered about $90 \mathrm{~min}$ before the end of surgery. The patient's body temperature was maintained over $35.8^{\circ} \mathrm{C}$ throughout the period of anesthesia. About 10 min before the end of surgery, infusion of remifentanil was stopped and $0.3 \mathrm{mg}$ ramosetron was administered to prevent postoperative nausea and vomiting. Only remifentanil was used during the surgery, and no other opioids were used before the patient regained consciousness. At a TOF count of 0 and post-tetanic count (PTC) of 10, 200 mg intravenous sugammadex (Bridion $^{\circledR}$, MSD, Korea) was administered. At that moment, the patient's BP was 90/50 $\mathrm{mmHg}$ and his HR was 60 beats/min. About 2 minutes later, sudden sinus bradycardia developed, and the patient's HR decreased to 29 beats/min, then spontaneously increased to 50 beats/min for $10 \mathrm{~s}$, but his BP remained normal (Fig. 1). After about $10 \mathrm{~s}$, the patient's HR returned to normal and all his vital signs were stable. The TOF count became 4 and the TOF ratio reached 0.2 , where it remained for $5 \mathrm{~min}$. Reasoning that the dose of sugammadex was inadequate, an additional dose of $200 \mathrm{mg}$ sugammadex was administered. About $30 \mathrm{~s}$ later, profound bradycardia (21-30 beats/min) developed again and hypotension $(60 / 40 \mathrm{mmHg})$ developed (Fig. 1). There were no signs of hypersensitivity reactions, such as urticaria, wheals, swelling, or increased airway pressure (mean airway pressure, $5.2 \mathrm{mmHg}$ ). Atropine $0.5 \mathrm{mg}$ was injected immediately, and his HR increased to 60 beats $/ \mathrm{min}$. However, his HR decreased to 21 beats/min again after $30 \mathrm{~s}$ and hypotension persisted. ABGA was done and the results were $\mathrm{pH}$ 7.341, $\mathrm{PaO}_{2} 272.1 \mathrm{mmHg}, \mathrm{PaCO}_{2} 33.8 \mathrm{mmHg}, \mathrm{SaO}_{2}$ 99.3\%, and $\mathrm{HCO}_{3} 19.0 \mathrm{mM}$. Profound bradycardia and hypotension occurred 4 more times at about $30 \mathrm{~s}$ time intervals. Ephedrine (10-20 mg) and phenylephrine (50-100 $\mu \mathrm{g}$ ) were administered intermittently to increase the patient's HR and BP. About 5 min later, his HR increased to over 70 beats/min. The TOF ratio became 0.9 about $10 \mathrm{~min}$ after administration of the additional sugammadex, and extubation was done after he recovered adequate self-respiration. He was moved to the recovery room, but hypotension alone developed again. Because of sustained hypotension (below $80 / 40 \mathrm{mmHg}$ ), he was transferred to the intensive care unit (ICU) and norepinephrine $0.03-0.05 \mu \mathrm{g} / \mathrm{min} / \mathrm{kg}$ was infused for 2 days. Cardiac examination was done postoperatively at the ICU. His cardiac enzymes were normal, and a postoperative ECG showed 1st degree AV block with QT prolongation (QT/QTc

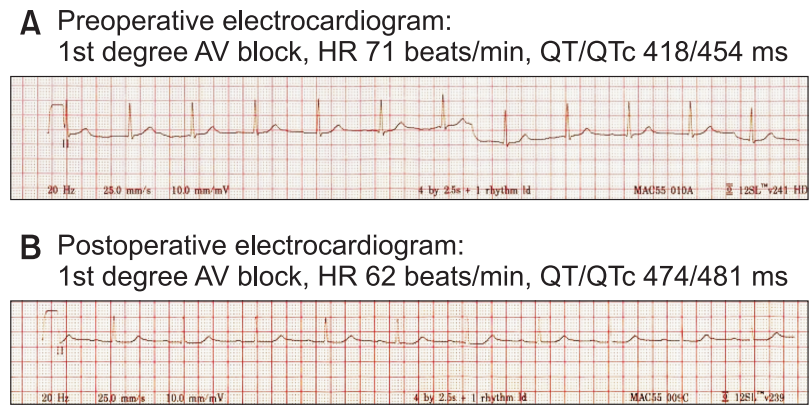

Fig. 2. Electrocardiogram of the patient. (A) Preoperative electrocardiogram showed 1st degree AV block (HR 71 beats/min, QT/QTc 418/454 ms). (B) After the use of sugammadex, the postoperative electrocardiogram showed QT prolongation (HR 62 beats/min, QT/QTc 474/481 ms). AV: atrioventricular, HR: heart rate, QTc: corrected QT.

418/454 ms; Fig. 2). Echocardiogram showed no significant abnormality. Holter ECG was assessed and showed no significant bradycardia. Beta-blocker medication was discontinued. The patient recovered normal BP, and was transferred to the ward. The patient was discharged in good condition on the 10th postoperative day.

\section{DISCUSSION}

Here we report on a patient who experienced profound bradycardia after the use of sugammadex. His HR decreased to 29 beats/min for 10 seconds temporarily after administration of $200 \mathrm{mg}$ sugammadex, and sustained bradycardia (2130 beats/min) and hypotension $(60 / 40 \mathrm{mmHg}$ ) developed after additional use of sugammadex. The hemodynamic response of the patient was very different from previous reported cases of anaphylaxis [1]. His airway pressure did not increase (mean airway pressure: $5.2 \mathrm{mmHg}$ ) and $\mathrm{BP}$ remained normal after the first use of sugammadex. This did not meet the diagnostic criteria for anaphylaxis that includes acute involvement of skin or mucosa with respiratory compromise and reduced BP [7]. Interestingly, bradycardia only lasted for $10 \mathrm{~s}$ after use of sugammadex. And only bradycardia and hypotension, without signs or symptoms of skin, mucosa, respiratory, or gastrointestinal organ involvement developed after use of additional sugammadex.

There are been several reports of cardiovascular changes associated with sugammadex administration. Recently, the FDA recommended that patients should be monitored closely for hemodynamic changes during and after use of sugammadex [2]. Prolongation of the QT interval was reported in 9 
patients after use of sugammadex [8], but no clear relationship between sugammadex and QTc prolongation was found in a subsequent randomized controlled study [9]. Seconddegree to third-degree AV block after the use of sugammadex has also been reported, but with no associated bradycardia $[10,11]$. The incidence of bradycardia associated with sugammadex has been reported to be approximately $1.9 \%$, and most cases have been clinically insignificant and effectively treated with anticholinergic agents [9]. However, severe bradycardia following the use of sugammadex was recently reported $[3,4,6]$.

Previous reports on mild bradycardia associated with sugammadex described temporary bradycardia without hypotension that recovered after administration of glycopyrrolate or atropine [5]. However, severe bradycardia with hypotension has also been reported recently. King et al. [12] reported severe bradycardia ( 26 beats $/ \mathrm{min}$ ) with hypotension ( $60 / 20$ $\mathrm{mmHg})$ after use of sugammadex $(2 \mathrm{mg} / \mathrm{kg})$ in a pediatric patient who received a liver transplant. His bradycardia and hypotension recovered after use of epinephrine. No clear mechanism was provided, but the authors speculated that concomitant administration of dexmedetomidine had an impact, even though the possibility were small. Sanoja and Toth [3] reported a case of severe bradycardia (35 beats/min) with hypotension within the first minute after administration of sugammadex, which further progressed to pulseless electrical activity arrest. Atropine was not effective for the patient, and cardiopulmonary resuscitation and epinephrine injection were done. The authors suspected that continuous infusion of ketamine and lidocaine during the surgery might have had a role in the dysrhythmia or subsequent arrest. According to these reports, concomitant administration of drugs that might affect the central sympathetic system could be the cause of severe bradycardia with hypotension, but this mechanism is unlikely in our case because bradycardia associated with sugammadex is not due to cholinergic effects [13]. However, in our case, the patient was only administered a $0.3 \mathrm{mg}$ bolus of ramosetron at the end of surgery, a drug not known to increase the QTc interval [14]. There was no exact evidence that ramosetron caused his 1st degree AV block, and no relationship between sugammadex and QTc prolongation was found in a randomized controlled study.

Interestingly, the patient in this case showed resistance to sugammadex. Shin et al. [6] reported a case similar to our case. They reported a case of severe bradycardia and hypotension after sugammadex injection, which improved after administration of atropine. In that case, neuromuscular blockade persisted, despite sugammadex administration and recovered after use of pyridostigmine. The authors hypothesized that factors, such as an inadequate dose of sugammadex, binding of sugammadex to other molecules instead of rocuronium, and hypothermia may have played roles. However, they concluded that these factors were unlikely causes, and the exact mechanism remains unknown. In our case, $200 \mathrm{mg}$ sugammadex was administered when the PTC was 10 and $200 \mathrm{mg}$ was administered again because the TOF ratio did not increase more than 0.2 for $5 \mathrm{~min}$. Despite the additional dose of sugammadex, the TOF ratio showed a slow increase and profound bradycardia and hypotension developed. It took about $10 \mathrm{~min}$ for the TOF ratio to become 0.9 after administration of additional sugammadex. The patient was almost normothermic $\left(35.8^{\circ} \mathrm{C}\right)$ and no drugs that could bind to sugammadex were administered during the anesthesia. In fact, the dose of sugammadex should be based on the actual body weight and status of neuromuscular blocks of the patient [15]. If spontaneous recovery of the twitch response reaches 1-2 PTCs, with no twitch responses to TOF, a dose of $4 \mathrm{mg} / \mathrm{kg}$ sugammadex should be used. In our case, the first dose of sugammadex should have been $300 \mathrm{mg}$, but we administered an inadequate dose. Further, we administered an additional $200 \mathrm{mg}$ dose of sugammadex after the first. That means $100 \mathrm{mg}$ of sugammadex was administered in excess of the appropriate dose. Administration of conventional reversal agents, such as neostigmine, instead of additional administration of sugammadex would have been better a better option for slow reversal of neuromuscular blockade or development of bradycardia after the use of sugammadex.

The mechanism of bradycardia associated with sugammadex is still unknown. However, it has been suggested that free sugammadex plasma molecules might be associated with the cardiovascular effects of sugammadex [3,4]. Excessive doses of sugammadex exceeding the recommended doses may provoke bradycardia or cardiac arrest. In our case, profound bradycardia and hypotension developed after use of an additional $200 \mathrm{mg}$ sugammadex, $100 \mathrm{mg}$ in excess of the recommended dose. Thus, the excessive dose of sugammadex would have played a role in the development of bradycardia and hypotension in our patient. Another possible mechanism 
may be related to preoperative conditions of a heart. The patient in our case had 1st degree AV block and borderline QT interval (QTc $454 \mathrm{~ms}$ ) and was taking a $\beta$-blocker for hypertension. After the use of sugammadex, slight QT prolongation (QTc $481 \mathrm{~ms}$ ) developed in our patient. Cardiac conditions, such as 1st degree AV block, QT prolongation and $\beta$-blocker medication, are not known to be associated with the development of bradycardia associated with sugammadex. As mentioned above, there is no clear relationship between sugammadex and QT prolongation [9], and there have been no reports about drug interactions between $\beta$-blockers and sugammadex. However, we cannot rule out that the patient's cardiac conditions played a role in the bradycardia because sugammadex has been reported to be somewhat related to prolongation of the QT interval [8].

Anticholinergic agents are still recommended for treatment of bradycardia associated with sugammadex [6]. However, epinephrine injection is recommended in severe and sustained bradycardia accompanied by hypotension in spite of use of anticholinergics $[3,4]$. In our case, we used atropine for the first bradycardia episode, which was effective, but we used ephedrine for the profound bradycardia with hypotension. Ephedrine was effective for bradycardia and hypotension, but the efficacy was temporary and limited. And the hypotension was sustained after the patient's HR recovered to normal. Thus, use of epinephrine from the beginning of bradycardia and hypotension would been a better choice.

In summary, we report a case of profound bradycardia with sustained hypotension in a patient who received sugammadex for reversal of neuromuscular blockade. We advise alertness to the possibly of bradycardia with hypotension associated with sugammadex, and close monitoring of patient hemodynamics and neuromuscular block level during the administration of sugammadex. And excessive doses of sugammadex should be avoided. Special attention should be paid to patients with slow recovery from neuromuscular blockade, in spite of administration of adequate doses of sugammadex. Conventional reversal agents could be better choices in these complicated cases.

\section{CONFLICTS OF INTEREST}

No potential conflict of interest relevant to this article was reported.

\section{ORCID}

Yong Jun Choi: https://orcid.org/0000-0002-2899-8429

Jeong Wook Park: https://orcid.org/0000-0002-3990-4715

Sang Hun Kim: https://orcid.org/0000-0003-3869-9470

\section{REFERENCES}

1. Godai K, Hasegawa-Moriyama M, Kuniyoshi T, Kakoi T, Ikoma K, Isowaki S, et al. Three cases of suspected sugammadex-induced hypersensitivity reactions. Br J Anaesth 2012; 109: 216-8.

2. Kim YH. Sugammadex: watch out for new side effects. Korean J Anesthesiol 2016; 69: 427-8.

3. Sanoja IA, Toth KS. Profound bradycardia and cardiac arrest after sugammadex administration in a previously healthy patient: a case report. A A Pract 2019; 12: 22-4.

4. Bhavani SS. Severe bradycardia and asystole after sugammadex. Br J Anaesth 2018; 121: 95-6.

5. Hunter JM, Naguib M. Sugammadex-induced bradycardia and asystole: how great is the risk? Br J Anaesth 2018; 121: 8-12.

6. Shin HS, Kim YR, Kim JA, Chung IS. Profound bradycardia and hypotension after sugammadex administration. J Clin Anesth Manag 2017; 2: 1-3.

7. Simons FE, Ardusso LR, Bilò MB, Dimov V, Ebisawa M, El-Gamal YM, et al. 2012 update: World Allergy Organization Guidelines for the assessment and management of anaphylaxis. Curr Opin Allergy Clin Immunol 2012; 12: 389-99.

8. Pühringer FK, Rex C, Sielenkämper AW, Claudius C, Larsen PB, Prins ME, et al. Reversal of profound, high-dose rocuronium-induced neuromuscular blockade by sugammadex at two different time points: an international, multicenter, randomized, dosefinding, safety assessor-blinded, phase II trial. Anesthesiology 2008; 109: 188-97.

9. Dahl V, Pendeville PE, Hollmann MW, Heier T, Abels EA, Blobner M. Safety and efficacy of sugammadex for the reversal of rocuronium-induced neuromuscular blockade in cardiac patients undergoing noncardiac surgery. Eur J Anaesthesiol 2009; 26: 874-84.

10. Osaka Y, Shimada N, Satou M, Masuda T, Ando T, Kozono Y, et al. A case of atrioventricular block (Wenckebach type) induced by sugammadex. J Anesth 2012; 26: 627-8.

11. Saito I, Osaka Y, Shimada M. Transient third-degree AV block following sugammadex. J Anesth 2015; 29: 641.

12. King A, Naguib A, Tobias JD. Bradycardia in a pediatric heart transplant recipient: is it the sugammadex? J Pediatr Pharmacol Ther 2017; 22: 378-81.

13. Booij LH, van Egmond J, Driessen JJ, de Boer HD. In vivo animal studies with sugammadex. Anaesthesia 2009; 64 Suppl 1: 38-44. 
14. Kim SH, Lee SM, Kim YK, Park SY, Lee JH, Cho SH, et al. Effects of prophylactic ramosetron and ondansetron on corrected QT interval during general anesthesia. J Clin Anesth 2014; 26: 511-6.
15. Lee S. What anesthesiologists ask to know and should know about the neuromuscular monitoring: an updated review. Anesth Pain Med 2017; 12: 1-8. 